\title{
The Small Satellite (CubeSat) Program as a Pedagogical Framework for the Undergraduate EE Curriculum
}

\section{W. Timothy Holman, Vanderbilt University}

Tim Holman received the B.S.E.E from the University of Tennessee and the M.S.E.E. and Ph.D.E.E. from the Georgia Institute of Technology. He is a member of the Institute for Space and Defense Electronics, and has held the position of Research Associate Professor in the Department of Electrical and Computer Engineering at Vanderbilt University since 2000. He specializes in the areas of low noise / low power analog, mixed-signal, and digital integrated circuit design, with a focus on radiation effects and radiationhardening-by-design techniques for high-performance analog and mixed-signal circuits.

\section{Brian David Sierawski, Vanderbilt University}

Brian Sierawski received his B.S.E in Computer Engineering in 2002 and M.S.E in Computer Science and Engineering in 2004 from the University of Michigan. He received his Ph.D. in Electrical Engineering in 2011 from Vanderbilt University. He has worked as a Research Engineer at the Institute for Space and Defense Electronics since 2005 and is currently an Adjunct Assistant Professor at Vanderbilt University.

\section{Robert Reed, Vanderbilt University}

Robert Reed is an expert on radiation effects in electronics. Robert has many years of experience in a variety of settings in spacecraft design and survivability and reliability. He has led programs in experimentation, modeling, and simulation of radiation effects in electronic systems. He has been involved with six separate space-based radiation effects experiments over the last 20 years: 1) RadFx-1,-2,-3: A series of CubeSat Based Radiation Effects Testbeds (PI), 2) Microelectronic and Photonics Test Bed (Instrument Card PI), and 3) Combined Release and Radiation Effects Satellite (Investigator), 4) Living With a Star Space Environment Testbed (mission definition and requirements). As a NASA civil servant, Robert was the lead radiation effects systems engineer for several NASA spaceflight projects, including the Hubble Space Telescope and the James Webb Space Telescope. He participated in development of MIL-STD-883, Test Method 1019 (Ionizing radiation (total dose) test procedure), development of the CRÈME-MC code that is commonly used by the satellite community, and other test guidelines used by DoD and NASA. He is very active in the radiation effects community, having served as General and Technical Chair of the IEEE Nuclear and Space Radiation Effects Conference and as a voting member of the IEEE Nuclear and Plasma Sciences Society Advisory Committee. He has own numerous awards for his work on spacecraft survivability and reliabilty: Three Outstanding Paper Awards at NSREC, IEEE Nuclear and Plasma Sciences Society Early Achievement Award, Clemson University Outstanding Young Alumni Award, NASA Best of the Best Achievement Awards. Robert is a fellow of the IEEE.

\section{Robert A. Weller, Vanderbilt University}

Andrew L Sternberg, Vanderbilt University

Andrew Sternberg received a B.S. degree in Engineering Science from David Lipscomb University in Nashville, TN in 1999. He received the M.S. degree in Electrical Engineering from Vanderbilt University, Nashville, TN in 2003, and the Ph.D. from Vanderbilt University in 2006. He joined the Vanderbilt University Institute for Space and Defense Electronics in March 2006.

\section{Rebekah Austin, Vanderbilt University}

Rebekah Austin is graduate student in Electrical Engineering at Vanderbilt University. Her research is in radiation effects on electronics and on how Vanderbilt's CubeSat program can be used in the undergraduate electrical engineering curriculum.

\section{Daniel M Fleetwood, Vanderbilt University}


Dan Fleetwood received B.S., M.S., and Ph.D. degrees from Purdue University in 1980, 1981, and 1984. He joined Sandia National Laboratories in 1984 as a Member of the Technical Staff. In 1990, he was named a Distinguished Member of the Technical Staff in the Radiation Technology and Assurance Department at Sandia. Dan accepted a position as Professor of Electrical Engineering at Vanderbilt University in 1999, and also holds a secondary appointment as Professor of Physics. In 2001-2003 he served as Associate Dean for Research in the School of Engineering. In 2003 he was named Chairman of Vanderbilt's Electrical Engineering and Computer Science Department, and in 2009 he was named Olin H. Landreth Chair in Engineering. Dan is author or co-author of more than 400 publications on radiation effects and low frequency noise, which have been cited more than 8800 times. He received the 2009 IEEE Nuclear and Plasma Sciences Merit Award. Dan is a member of ASEE and a Fellow of the IEEE and the American Physical Society. 


\section{The Small Satellite (CubeSat) Program as a Pedagogical Framework for the Undergraduate EE Curriculum}

\section{Abstract}

The small satellite (CubeSat) concept provides a means for Vanderbilt University students to become directly involved in the design and launch of space systems. In this paper, we illustrate how our small satellite research program is being used to introduce fundamental circuit design concepts to undergraduate electrical engineering (EE) courses, and to reinforce student comprehension of their use in modern integrated systems.

\section{Introduction}

The development of new techniques to improve the effectiveness of engineering laboratories and project courses remains an ongoing focus of research in engineering education [1-11]. The Vanderbilt University CubeSat program provides a particularly compelling framework for introducing new hardware and software concepts into undergraduate laboratories and introductory engineering courses. Space systems, by their very nature, incorporate subsystems for RF communications, power regulation and management, environmental control, digital signal processing, microprocessor control, data conversion, and remote sensing (among others) into a single package.

CubeSats are a class of small satellites that are compact, measuring $10 \times 10 \times 10 \mathrm{~cm}$ for one unit (1U), and built from low-cost commercial off-the-shelf components. They operate as independent spacecraft performing earth and space science with the goal of one- to two-year operational lifetimes. Consequently, CubeSats provide an excellent means of capturing students' imaginations while familiarizing them with the fundamental techniques behind these subsystems.

The CubeSat concept provides a pedagogical framework that gives students an introduction to circuits (and circuit concepts) that would not normally be explored at the freshman or sophomore level. For example:

(1) Satellite RF communication systems can provide a basis for experiments in oscillators, modulation techniques, and simple transmitters and receivers.

(2) Satellite power management systems can provide a basis for experiments in high-current output buffers, voltage regulators, and power supply design.

(3) Satellite sensors and data acquisition systems can provide a framework for investigations into data transmission via infrared diodes, and the design of simple analog-to-digital (A/D) and digital-to-analog (D/A) converters.

(4) Environmental control systems can provide an introduction to comparators, hysteresis (positive feedback), multivibrator circuits, and sensors. 
The CubeSat curriculum is intended to introduce these types of circuit applications while reinforcing student retention and comprehension. For example, a student might first learn about data converters as a freshman, and interface $\mathrm{A} / \mathrm{D}$ and $\mathrm{D} / \mathrm{A}$ converter ICs with a simple digital circuit in an introductory digital systems class. As a sophomore, (s)he might use operational amplifiers and comparators to construct some basic A/D and D/A circuit topologies (e.g. flash and single-slope converters) in a laboratory course. Later, as a junior (s)he might build a currentsumming D/A converter using MOS and bipolar transistors. In each case, the CubeSat program would provide a pedagogical framework as the student learns about data converters in a "top down" manner, with relevant documentation serving to reinforce the presentation in each succeeding course.

In this paper we will describe how various elements and techniques from our CubeSat program are currently being used to enhance the "hands-on" aspects of the undergraduate EE curriculum, and provide students with a more comprehensive and relevant exposure to modern design concepts. The following is not a comprehensive description of the full scope of the intended CubeSat enhancements to our EE curriculum, but does serve to show two possible examples of how CubeSat pedagogy can be applied to introductory engineering courses.

\section{Introduction to Engineering}

Introduction to Engineering is a first-year course designed to expose students to multiple engineering disciplines through the selection of three different course modules during the semester. Each module lasts five weeks and provides students with adequate background in each discipline to accomplish a meaningful task. Team-based projects are developed around topics in Biomedical Engineering, Chemical and Biomolecular Engineering, Civil and Environmental Engineering, Computer Engineering, Computer Science, Electrical Engineering, and Mechanical Engineering. The modules are also a factor in each department's undergraduate enrollment, as they provide a means to assist undecided engineering students in choosing a major field.

Previously, the Electrical Engineering module required students to construct a mobile robot kit. Once the kit was completed and tested, students would compete to race their robots against other teams. Although students enjoyed the competitive aspect of the module, the robot kits did not allow for any significant hardware/software co-design or customization, which is integral to modern EE systems and applications. In addition, the robot kit module was a standalone experience with no direct follow-up in our core EE curriculum until the senior year.

In the new EE module, students prototype portions of a small satellite using an Arduino development board as an electronics platform. The Arduino Uno board and its associated shields (printed circuit boards that extend the capabilities of the Arduino) are well-suited to the size constraints encountered in small satellite design. The extensibility of the platform and the wellsupported development environment make a variety of design topics accessible at the first-year level. Most students entering the course have little or no previous experience with controller programming software, but find the simple examples provided in this module to be sufficient. The peripheral support in the microcontroller allows for experiments with sensors, remote actuators, and wireless communications within a single class section. With this platform the students can achieve relatively high-level design goals that would have been impossible for first- 
year students to contemplate just a decade earlier. The EE module introduces students to simple circuits including analog and digital signals, and enables them to make meaningful measurements of the physical environment and communicate the results. More importantly, CubeSat concepts introduced in the EE module can be revisited in the sophomore and junior years as EE students progress through the core curriculum. This prepares them for a mature senior capstone experience in either actual CubeSat design and construction or other areas.

Students are organized into groups of three or four and assume rotating roles of hardware, software, science, and operational leads. This organization provides structure within each group as the students learn to use the hardware to perform higher-level tasks. Each group performs eight experiments and one final demonstration.

In the first experiment, students learn to (1) identify resistor values using color bands, (2) breadboard simple resistor circuits powered by the Arduino USB port, (3) use a multimeter to measure resistance, voltage, and current, and (4) identify open and short circuits. In the second experiment, students are introduced to the microcontroller using a pushbutton and light-emitting (LED) diode to serve as digital inputs and outputs. Students construct the provided circuit and upload example code to the microcontroller to sample the pushbutton input and drive the LED output. Using their developing measurement skills, they relate the discrete voltage levels of the Arduino I/O to the digital values in their software. Finally the microcontroller code is modified to alter the blink rate of the LED, thereby introducing the concepts of frequency and duty cycle in periodic signals.

In the third and fourth experiments the students are provided with analog sensors, and must connect these components to the Arduino microcontroller to make measurements of temperature and luminance. The first of these experiments begins by exploring how to measure a voltage with the microcontroller analog-to-digital converter, and return the measurement to a laptop computer. The development environment makes this a simple process and allows students to focus on the act of making the measurement. The students then incorporate a thermistor into the design and extract pertinent information from a datasheet regarding the temperature-voltage relationship in order to convert the measurement to degrees Celsius within the microcontroller.

In the next session the students construct circuits to measure luminance and infrared radiant intensity. This experiment introduces NPN phototransistors as optical detectors. Students must again examine a datasheet to determine the spectral sensitivity and measured luminance/intensity. This experiment provides a further opportunity to relate frequency and the wavelength of an electromagnetic signal.

In the fifth experiment, students construct a common-emitter circuit to demonstrate the amplifying and switching behavior of an NPN bipolar junction transistor. The students bias the circuit into the cutoff, forward-active, and saturation regions to measure currents and report the current gain. With additional measurements of voltage, students learn how a transistor can be used as a switch for digital signals.

The sixth experiment requires the students to make a measurement of the ambient magnetic field. The groups receive a 3-axis magnetometer and must use the inter-integrated circuit $\left(\mathrm{I}^{2} \mathrm{C}\right)$ 
communication protocol to obtain a measurement. They learn how a binary string is transmitted over a serial interface, and then use the microcontroller to configure the magnetometer. After proper configuration and sampling the sensor, the students validate their reading of the magnetic field intensity against the NOAA Maps of Magnetic Elements [12].

In the seventh experiment, the students apply the concepts of frequency and duty cycle to investigate how pulse-width modulation can control a servo. The students command the microcontroller to output various pulse widths and actuate the servo to predefined positions.

The eighth and final structured experiment eliminates the power provided by the USB connection and adds a Li-ion battery to operate the prototype independently. Students add an adjustable voltage regulator and provide an additional $1.8 \mathrm{~V}$ source to the prototype by designing an appropriate resistor divider network.

In the final two sessions of the course, the groups are given an unstructured request to build a system to measure temperature and light and magnetic field strength, and transmit the results via radio to a base station while operating independently of external power or command. Groups are provided with Xbee-PRO $900 \mathrm{MHz}$ radios for wireless data transmission. After meeting the stated requirements, each group must provide the microcontroller source code and circuit schematic diagram detailing its design. Each group broadcasts its data on a separate RF channel, with all incoming data displayed real-time on an overhead projector. While this is not a formal competition, the students take considerable pride in seeing their data received and displayed in a real-time manner.

\section{Circuits II laboratory}

For EE majors, Circuits II is normally taken in the second semester of the sophomore year. This course introduces concepts such as Laplace transforms, frequency response, Bode plots, and passive/active filter design. By this point in the EE curriculum, students have learned about passive circuit elements (resistors, capacitor, inductors) and operational amplifiers. The laboratory for Circuits II provides them with their first experience in constructing electronic circuits using these components.

In previous semesters, the Circuits II laboratory was strongly coupled to (and limited by) the topics presented in the class lectures. Consequently, students spent the entire semester constructing standard op amp circuits (summing amplifiers, differential amplifiers, etc.) and various types of active filter circuits. These experiments tended to be somewhat repetitive in nature, and were not associated with "real world" applications in any clear manner.

The new CubeSat experiments take the laboratory experience in a very different direction. The original laboratory experiments have been revised and condensed into the first half of the semester. In the second half of the semester, five new CubeSat experiments are presented. Each of these experiments builds upon ideas first introduced in Introduction to Engineering, and is designed to introduce students to a concept in circuit design that is relevant to space systems in particular, and to more modern electronics systems in general. Headphones and light emitting diodes (LEDs) provide audio and visual feedback in order to encourage and maintain student 
interest. Each circuit is designed to be relatively simple to construct, while simultaneously providing as broad a range of experimental insight as possible.
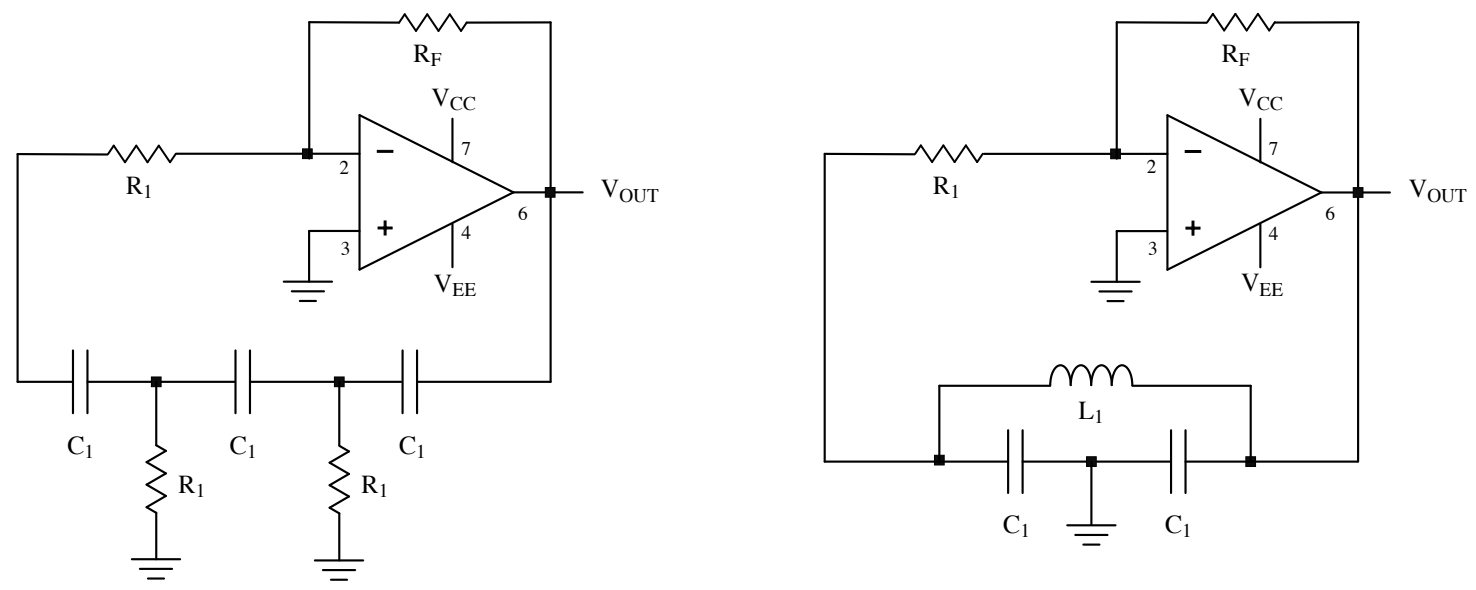

Fig. 1. Phase-shift and Colpitts oscillator circuits for generating sinusoidal waveforms.

In the first CubeSat experiment, students build simple oscillator circuits (Fig. 1) and learn how sinusoidal signals can be generated. By opening and closing the feedback loop, they create a $\mathrm{CW}$ (continuous wave) modulated signal, and learn about Morse code. Then, by connecting and disconnecting an additional capacitor in parallel with a capacitor in the phase-shift oscillator, students learn about PSK (phase shift keying) modulation.

In the second CubeSat experiment, students learn how to use an op amp as a comparator, and how positive feedback adds hysteresis to the output response. Next, they learn how square waves (e.g., clocks for digital systems) can be generated with an astable multivibrator (Fig. 2).

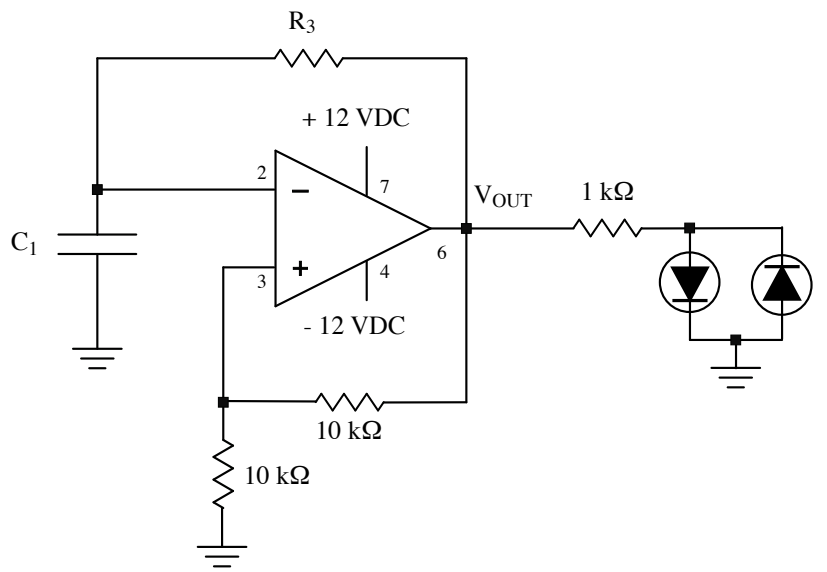

Fig. 2. Astable multivibrator circuit for generating square waves.

The bistable multivibrator in Fig. 3 shows students how a data bit can be stored and flipped from one state to another by touching the input wire to the positive or negative power supply. Using this multivibrator design, students learn the fundamental concept behind memory circuits. 


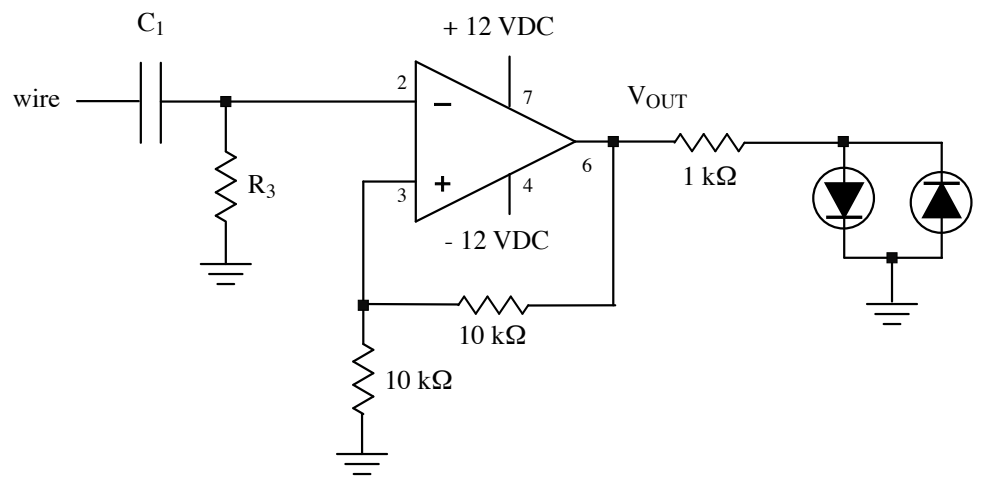

Fig. 3. Bistable multivibrator for demonstrating the concept of a memory circuit.

The third CubeSat experiment introduces students to analog-to-digital and digital-to-analog conversion. The 2-bit flash A/D converter in Fig. 4 builds upon the use of op amps as comparators, while the 2-bit summing D/A converter in Fig. 5 is a modification of a summing amplifier that was constructed in the first half of the semester.

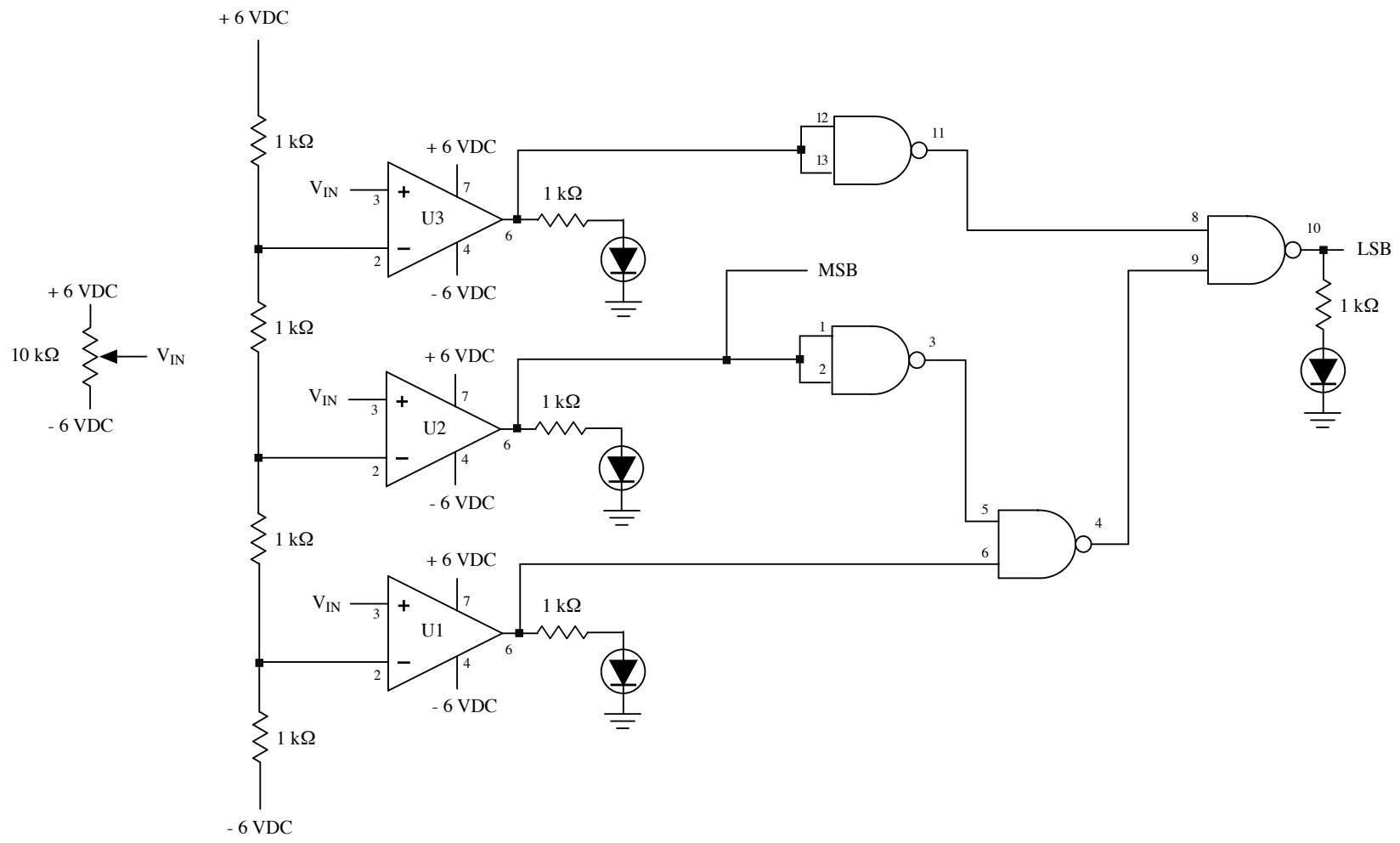

Fig. 4. A 2-bit flash analog-to-digital converter using op amps and CMOS digital logic. 


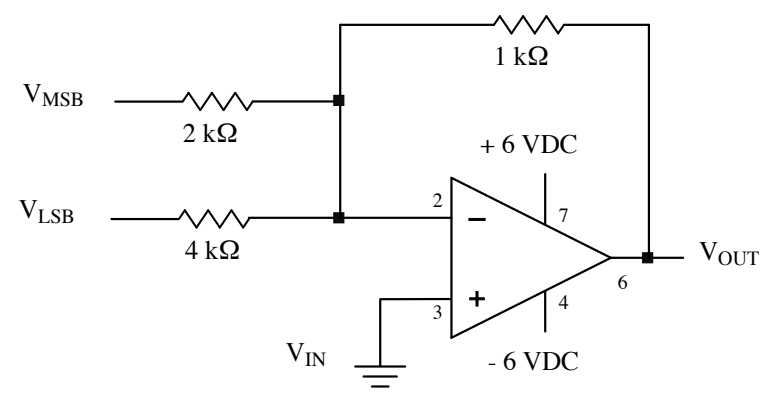

Fig. 5. A 2-bit digital-to-analog converter based on a summing amplifier.

In the fourth CubeSat experiment, students construct a simple optical transmitter using an infrared (IR) diode, and a simple optical receiver using an IR phototransistor (Fig. 6). To make the lab more interesting, a transistor radio is used as an audio source, while the optical path between the diode and phototransistor can be blocked and unblocked using a sheet of paper. Students are shown that these two circuits can be used either to transmit data or to sense when an object passes near an optical detector.
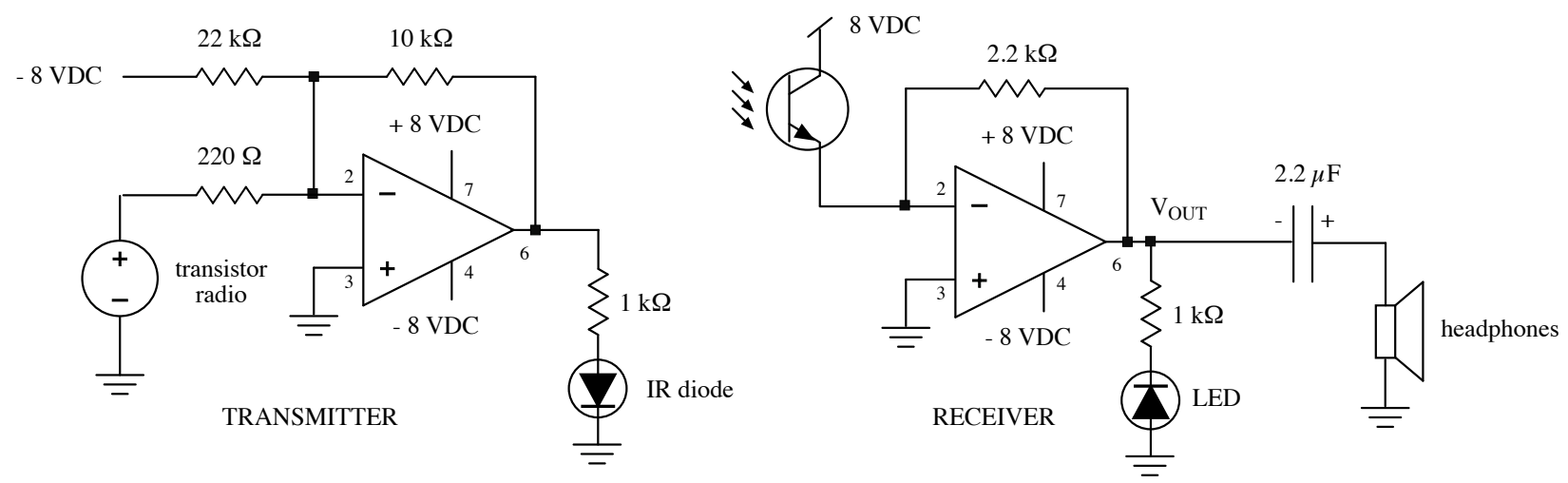

Fig. 6. An infrared communications link for transmitting an audio signal.

In the final CubeSat experiment, students construct an AM (amplitude modulated) radio transmitter (Fig. 7) using a microphone and preamplifier connected to a mixer circuit made from an LT1228 current feedback amplifier [13]. Although the transmitter range is limited to a few inches because of the very low output power of the LT1228 integrated circuit, students can combine the techniques of sinusoidal carrier generation, microphone preamplification, and AM modulation to hear their voices over a nearby transistor radio. Finally, by positioning the microphone next to the speaker of the radio, students hear the classic "squeal" of positive feedback, which returns them to the phase shift oscillation concept of the first CubeSat experiment. 


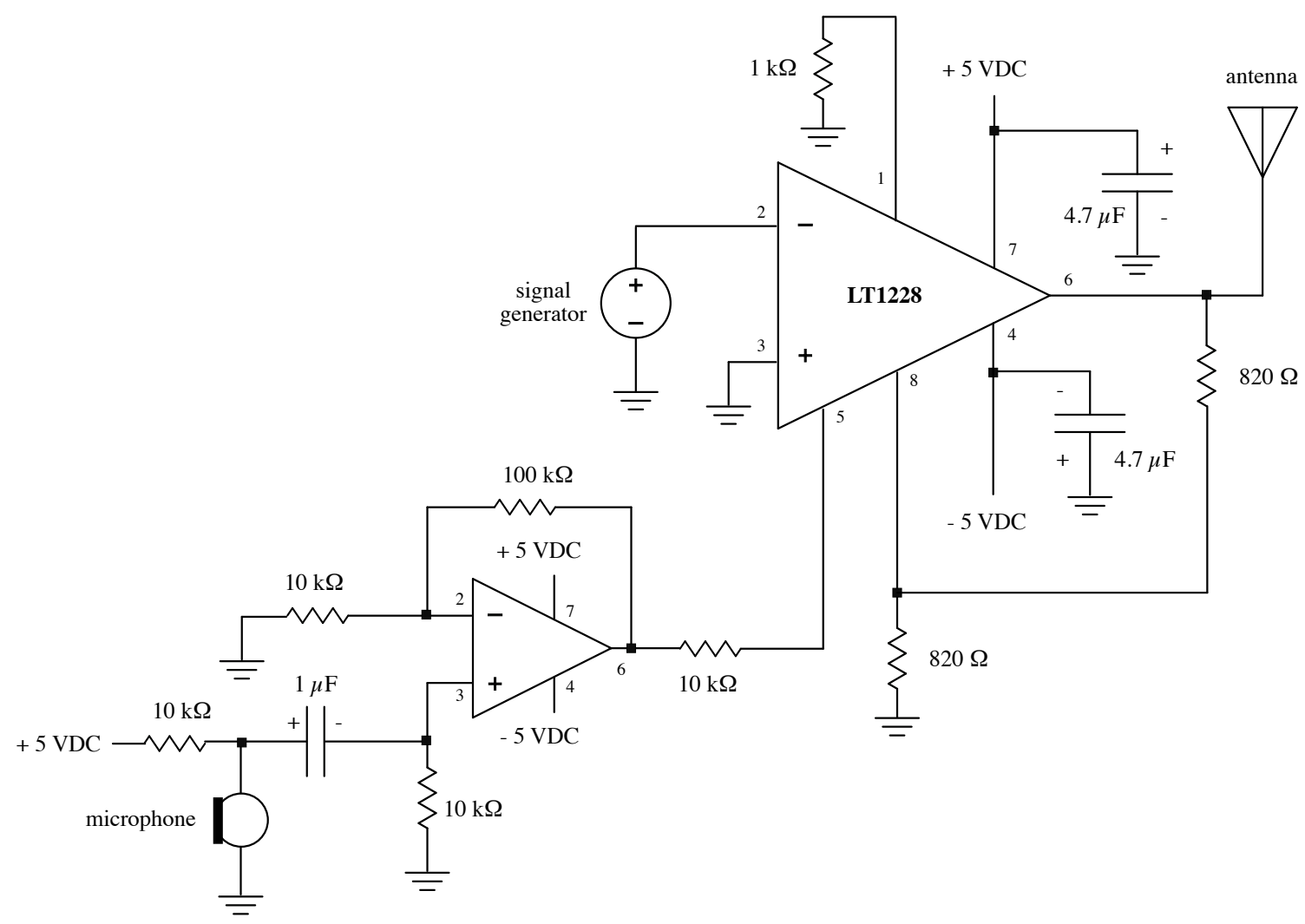

Fig. 7. A simple AM transmitter constructed using a microphone and a current feedback amplifier.

\section{Student feedback}

Initial student feedback to the incorporation of CubeSat material into the core EE curriculum has been quite favorable. Course evaluations for Introduction to Engineering show the course material is accessible to first-year students, with students reporting above average levels of learning and intellectual challenge. Student evaluations for the Circuits II laboratory were compared for the Fall 2012 and Fall 2013 semesters, during which the transition between the old and new curricula took place. Table 1 summarizes student responses to three questions from the end-of-semester course evaluations. While not conclusive, the results for 2013 are suggestive of improved outcomes for students. We note that these sophomores took the old EE module on robotics. Next year will be the first time that students who have taken the CubeSat module in Introduction to Engineering will experience the revised Circuits II laboratories, which will give us an opportunity to compare the effectiveness of the changes with a more prepared cohort.

\section{Next stages}

With the introduction of CubeSat concepts to first-year students and the increasing depth of treatment of circuit concepts in our Circuits II laboratory, the next phase of integration is to build on this framework to further enhance our first-semester junior, required Electronics I laboratory. Students will construct a transistor-based circuit that will be interfaced with a CubeSat circuit board. Plans are being developed to incorporate new laboratory modules in this course that will 
serve as a bridge from our core required circuits and electronics courses to the senior capstone experience.

\begin{tabular}{|l|c|c|c|c|}
\hline \multirow{2}{*}{ Question } & \multicolumn{2}{|c|}{2012 (32 responses) } & 2013 (29 responses) \\
\cline { 2 - 5 } & Average & Std. Dev. & Average & Std. Dev. \\
\hline $\begin{array}{l}\text { Rate the course requirements compared } \\
\text { to others you have taken. } \\
(1=\text { very easy, 5 = most rigorous) }\end{array}$ & 2.62 & 0.64 & 3.03 & 0.55 \\
\hline $\begin{array}{l}\text { Estimate how much you learned in the } \\
\text { course. } \\
\begin{array}{l}(1=\text { almost nothing, 5 = considerably } \\
\text { above average) }\end{array}\end{array}$ & 3.15 & 0.93 & 3.27 & 0.82 \\
\hline $\begin{array}{l}\text { Rate the effectiveness of this course in } \\
\text { challenging you intellectually. } \\
(1=\text { poor, 5=excellent })\end{array}$ & 3.12 & 0.78 & 3.34 & 0.54 \\
\hline
\end{tabular}

Table 1. Comparison of student evaluations for the Circuits II laboratory.

We will also be developing more comprehensive assessment metrics to evaluate the effectiveness of the CubeSat modules in enhancing the undergraduate EE experience. The promotion of this program has already led to a surge of student interest in undergraduate research and independent study courses, and an increase in the number of students obtaining amateur radio licenses. This provides a pool of talent from which to draw to develop significant capstone design experiences based around not only the CubeSat platform, but other areas involving microelectronics and signal processing applications.

\section{Conclusion}

Given the favorable student response to date, future enhancements are already being planned, including the expansion of the CubeSat pedagogy into other EE core courses and the introduction of actual CubeSat hardware into the laboratory experience. Future Introduction to Engineering modules will culminate in a student design competition that revolves around some customizable aspect of CubeSat hardware and software design, e.g. building an attitude detection sub-system that determines a CubeSat's orientation in space. Overall, the response to the CubeSat research program among students and instructors has been very favorable. We expect that the CubeSat pedagogy will have a significant and lasting benefit on student outcomes as it is incorporated into more core EE classes and laboratories at Vanderbilt University.

\section{Bibliography}

[1] Feisel, Lyle D., and Albert J. Rosa. "The role of the laboratory in undergraduate engineering education." Journal of Engineering Education 94, no. 1 (2005): 121-130.

[2] Roppel, Thaddeus A., John Y. Hung, Stuart W. Wentworth, and A. Scott Edward Hodel. "An interdisciplinary laboratory sequence in electrical and computer engineering: Curriculum design and assessment results." Education, IEEE Transactions on 43, no. 2 (2000): 143-152. 
[3] Edward, Norrie S. "The role of laboratory work in engineering education: student and staff perceptions." International Journal of Electrical Engineering Education 39, no. 1 (2002): 11-19.

[4] Karadimas, Dimitris, and Kostas Efstathiou. "Design, implementation and evaluation of a remote laboratory system for electrical engineering courses." In Advanced Learning Technologies, 2006. Sixth International Conference on, pp. 408-412. IEEE, 2006.

[5] Campbell, J. Olin, John R. Bourne, Pieter J. Mosterman, and Arthur J. Brodersen. "The effectiveness of learning simulations for electronic laboratories." Journal of Engineering Education 91, no. 1 (2002): 81-87.

[6] Gillet, D., H. A. Latchman, Ch Salzmann, and O. D. Crisalle. "Hands-On Laboratory Experiments in Flexible and Distance Learning." Journal of Engineering Education 90, no. 2 (2001): 187-191.

[7] Gustavsson, Ingvar, Kristian Nilsson, Johan Zackrisson, Javier Garcia-Zubia, Unai Hernandez-Jayo, Andrew Nafalski, Zorica Nedic et al. "On objectives of instructional laboratories, individual assessment, and use of collaborative remote laboratories." Learning Technologies, IEEE Transactions on 2, no. 4 (2009): 263-274.

[8] Goodwin, Graham C., Adrian M. Medioli, Willy Sher, Ljubo B. Vlacic, and James S. Welsh. "Emulation-based virtual laboratories: a low-cost alternative to physical experiments in control engineering education." Education, IEEE Transactions on 54, no. 1 (2011): 48-55.

[9] Cagiltay, Nergiz Ercil, Elif Aydin, Rusen Oktem, Ali Kara, Marian Alexandru, and Bodo Reiner. "Requirements for remote RF laboratory applications: An educators' perspective." Education, IEEE Transactions on 52, no. 1 (2009): 75-81.

[10] Rojko, Andreja, Darko Hercog, and Karel Jezernik. "Power engineering and motion control web laboratory: Design, implementation, and evaluation of mechatronics course." Industrial Electronics, IEEE Transactions on 57, no. 10 (2010): 3343-3354.

[11] Chu, Rui Hong, DD-C. Lu, and Swamidoss Sathiakumar. "Project-based lab teaching for power electronics and drives." Education, IEEE Transactions on 51, no. 1 (2008): 108-113.

[12] NOAA National Geophysical Data Center. (2010) [Illustration of main field intensity] Maps of Magnetic Elements from the WMM 2010. Retrieved from http://www.ngdc.noaa.gov/geomag/WMM/image.shtml

[13]LT1228 datasheet by Linear Technology. Retrieved from http://cds.linear.com/docs/en/datasheet/1028fb.pdf

[14] Franco, S., Design with operational amplifiers and analog integrated circuits, ISBN 9780072320848 , McGraw-Hill, 2002. 